\title{
Case Study: Converting Paper-based Case Report Forms to an Electronic Format (e-CRF) with ACASI Self-Report Integration
}

\author{
Stan Mierzwa' ${ }^{1}$, Samir Souidi ${ }^{1}$, Carolyne Akello ${ }^{2}$, Juliane Etima ${ }^{2}$, Richard Ssebagala ${ }^{2}$, Monica \\ Nolan², Samuel Kabwigu ${ }^{2}$, Clemensia Nakablito \\ 1. Information Technology, Population Council, New York, USA
}

2. Makerere University - Johns Hopkins University (MU-JHU) Research Collaboration CRS, Kampala Uganda

\begin{abstract}
This paper will discuss the integration of electronic Case Report Forms (e-CRFs) into an already existing Android-based Audio Computer-Assisted Self-Interview (ACASI) software solution that was developed for a public health project in Kampala, Uganda, the technical outcome results, and lessons learned that may be useful to other projects requiring or considering such a technology solution. The developed product can function without a connection to the Internet and allows for synchronizing collected data once connectivity is possible. Previously, only paper-based CRFs were utilized at the Uganda project site. A subset or select group of CRFs were targeted for integration with ACASI in order to test feasibility and success. Survey volume, error rate, and acceptance of the system, as well as the operational and technical design of the solution, will be discussed.

Keywords: Self-Report Data Collection; Case Report Forms; CRF; e-CRF; Tablets; Android; Clinical trials; Feature integration

Correspondence: smierzwa3@gmail.com

DOI: $10.5210 /$ ojphi.v9i3.7929

Copyright @2017 the author(s)

This is an Open Access article. Authors own copyright of their articles appearing in the Online Journal of Public Health Informatics. Readers may copy articles without permission of the copyright owner(s), as long as the author and OJPHI are acknowledged in the copy and the copy is used for educational, not-for-profit purposes.
\end{abstract}

\section{Introduction}

An informal literature review of journal articles found in Google Scholar indicate a lack of samples or experiences in public health or clinical trials research in which paper case report forms were integrated with Audio Computer-Assisted Self-Interview (ACASI) and Computer-Assisted Personal-Interview (CAPI) type of questionnaires. Case report forms (CRFs) are designed to record data on each trial subject during the course of a clinical trial as defined by the protocol. A CRF for each patient in the study must be completed and signed by the investigator and assessor. All of the events that happen in the clinical trial should be fully documented, including adverse reactions. Within clinical research the CRF can include a questionnaire used to collect data on such 
items as adherence and acceptability on participants, which in many cases warrant the use of a more private self-report strategy.

Electronic data collection in rural settings in sub-Saharan Africa with mobile devices has been found to be superior to that of a paper-based system, in regards to accuracy and completeness of data. A comparison of paper-based data collection with electronic data collection showed that direct electronic data entry was faster and $25 \%$ cheaper. Data was more accurate (7\% paper-based error rate versus $1 \%$ electronic error rate) and omission did not occur with electronic data collection. Delayed data turnaround times and late error detections in the paper-based system which made error corrections difficult were avoided using electronic data collection [1]. In many behavioral and adherence and acceptability research studies there could be a need to include ACASI survey technology, in order to provide the participants with more confidentiality in answering very sensitive, personal or culturally taboo questions. Such sensitive ACASI surveys have been performed outside the technology solutions provided for e-CRF systems. In the Hi-4TU (Health Improvements-For-Teen Ugandans) effort the Population Council's customizable ACASI \& CAPI solution was integrated with the pilot e-CRF demographic and screening forms so that all the data collected would be centralized in one electronic data source. The benefit of using one single solution are many, but one obvious advantage is that data integration or merging between ACASI, CAPI and e-CRF would not be required, thus eliminating a more complicated data consolidation step which can introduce errors.

\section{Research Project Background}

The specific research project discussed in this paper is called "Health Improvements-For-Teen Ugandans" or HI-4-TU study. It includes a study population of 519 currently pregnant adolescents (without diagnosed pregnancy related complications), who are attending antenatal care in Kampala, Uganda and are aged 15 to 19 at enrollment. The study design is a randomized controlled study with the objective of testing the acceptability and effectiveness of two enhanced peer led, reproductive health interventions as compared with routine health care. The behavioral interventions included enhanced group support and enhanced individual support. The study participants are individually randomized to one of three arms. Electronic data collection commenced in March 2016 and is scheduled to be completed in October 2017.

The collaboration in the research project was between information technology engineers and specialists at the Population Council and HIV and STI researchers at the Makerere University Johns Hopkins University (MUJHU) site in Kampala, Uganda. Further study information is published on the ClinicalTrials.Gov web portal.

\section{Methods}

The base technology utilized in the project was the Population Council's customized ACASI/CAPI solution. The PC ACASI/CAPI, otherwise known as SuveyX, solution was programmed and modified to support two individual CRFs that were required by the site. At the outset, the research team was interested in integrating the entire set of CRFs, which totaled 12, but since this was the first attempt at integrating CRFs with the PC ACASI/CAPI solution it was decided to pilot with two existing CRFs as a way to test feasibility of such a system. The MUJHU site was familiar with 
implementing the PC ACASI solution after several years' experience participating in research in collaboration with an earlier research effort, for which the Population Council and MUJHU were involved and using the already existing ACASI solution. This previous experience and our established collaboration helped move this pilot approach forward.

The MUJHU site provided the finalized CRF and ACASI surveys to the Information Technology staff at PC and this allowed us to start the design, architecture and technical build out of the solution. During the time the electronic instrument was being built out, the MUJHU site simultaneously conducted the necessary translations to the Bantu family language of Luganda and recorded the .MP3 audio files required for ACASI. MPEG-1 or MPEG-2 audio layer III is the audio coding format commonly referred to as .MP3. Pre-tests without the use of participants were performed at the site with research staff. The pre-testing uncovered translation and audio file clarity problems which were resolved by working closely with the US based Population Council staff. After corrections were made to the electronic instrument the Uganda research staff was notified to perform the automatic update on each of the tablet devices. The software update process was initiated by pressing an update button on an administrative screen on each device, which in turn automatically connected to a custom developed Microsoft Azure cloud service to determine the program and database differences between the device and the most recent published technology solution.

\section{ACASI + CRF Designed Solution - Operational}

Each of the Android tablet units used for the CRF and ACASI data collection system is configured identically with the software solution. Each unit is however named individually to help distinguish on which device data is collected, and the unique assigned tablet name is saved with the result data as it is collected. In addition, as seen in Figure 1, each CRF and ACASI system administrator that will establish the electronic survey for participants is assigned a unique ID that is to be utilized when starting up the application. The unique ID is saved with each record created in the solution.

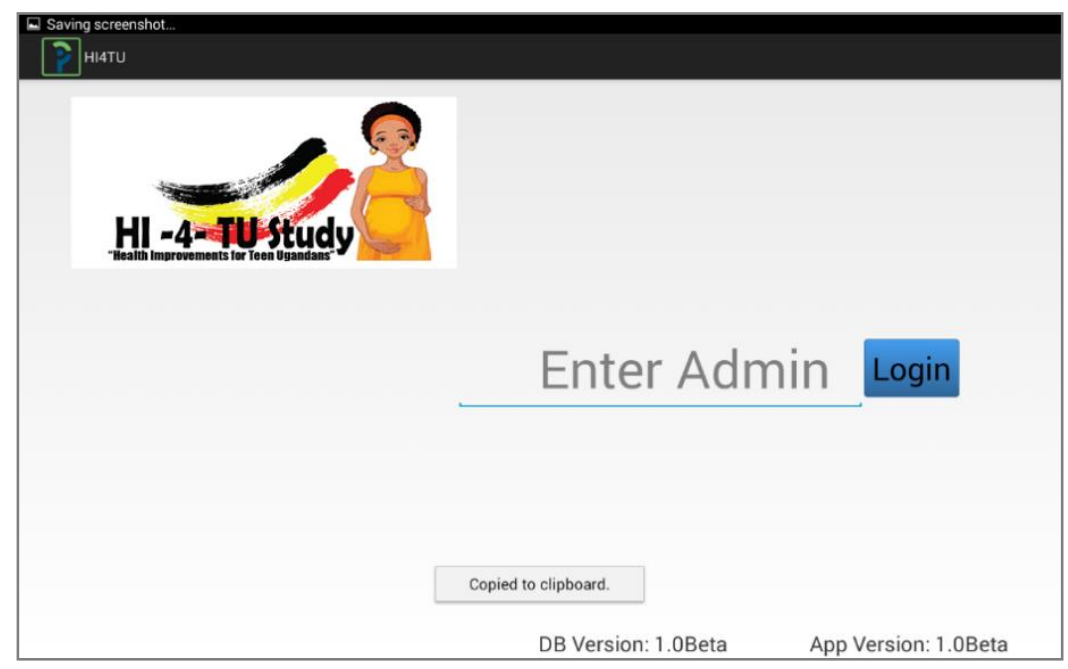

Fig. 1. Entry screen to the HI - 4 - TU ACASI/CRF solution. 


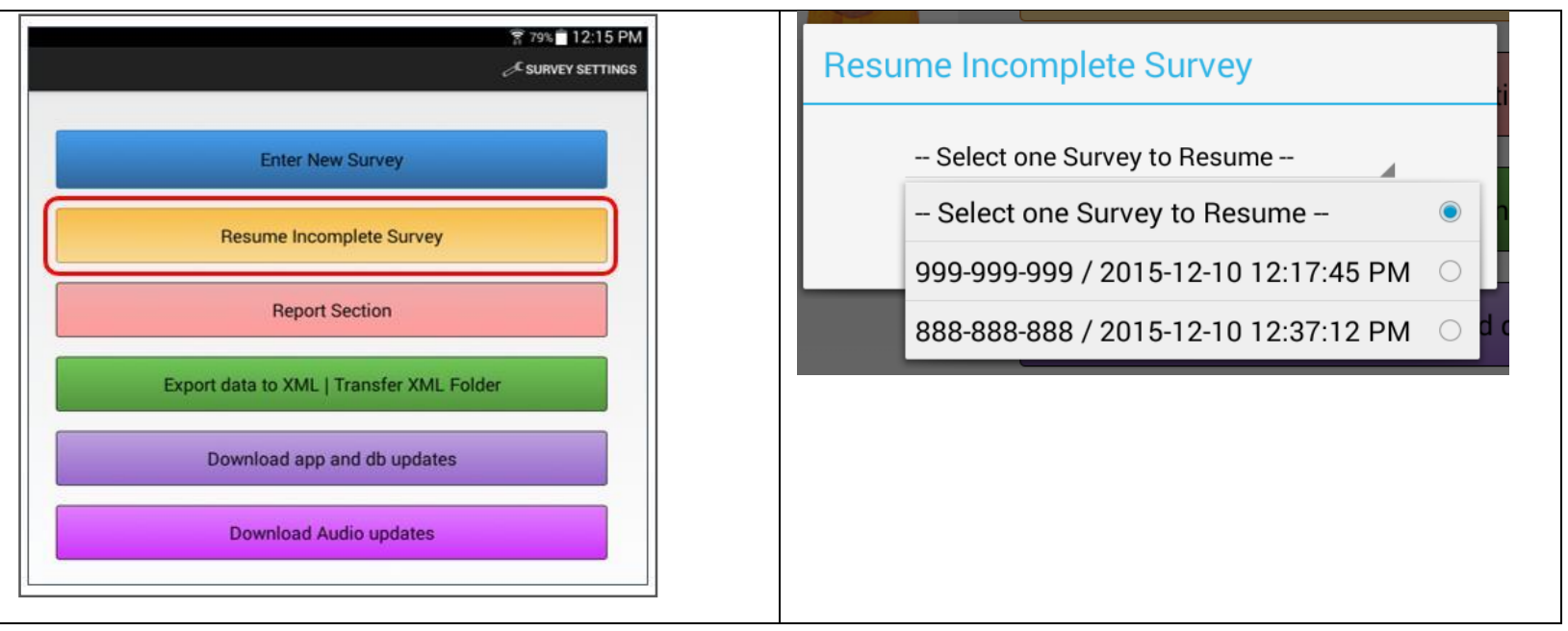

Fig. 2. (a) Administrative choices (b) Ability to resume a CRF or ACASI survey.

The administrator may resume incomplete surveys and also view all of the surveys completed on the specific device, as shown in Figure 2.

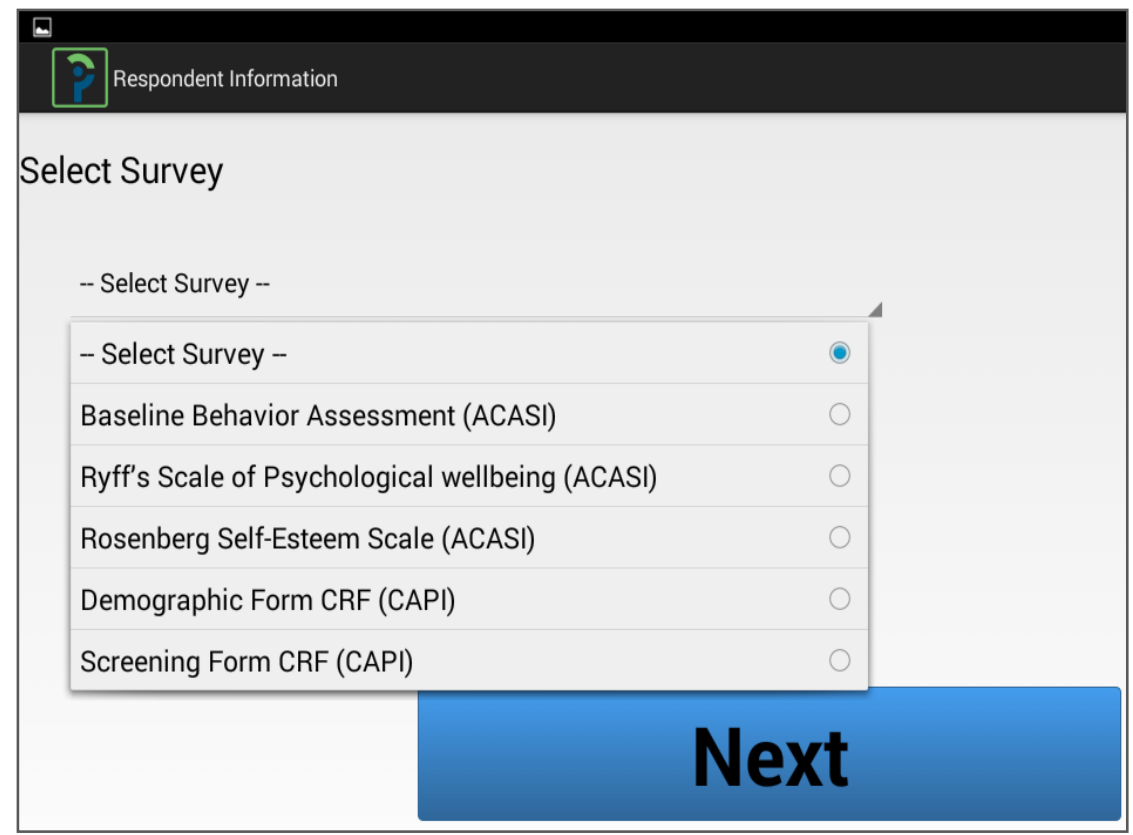

Fig. 3 Survey choices included 3 ACASI and 2 CRF options.

The overall Hi-4-TU randomized control trial included more CRFs than those that were presented in the electronic tablet solution discussed in this paper. As part of this pilot effort, two CRFs were selected by the MUJHU site to be part of this electronic tablet data collection system. These forms are included on the list of options in Figure 3, namely the Demographic Form CRF and the Screening Form CRF. 


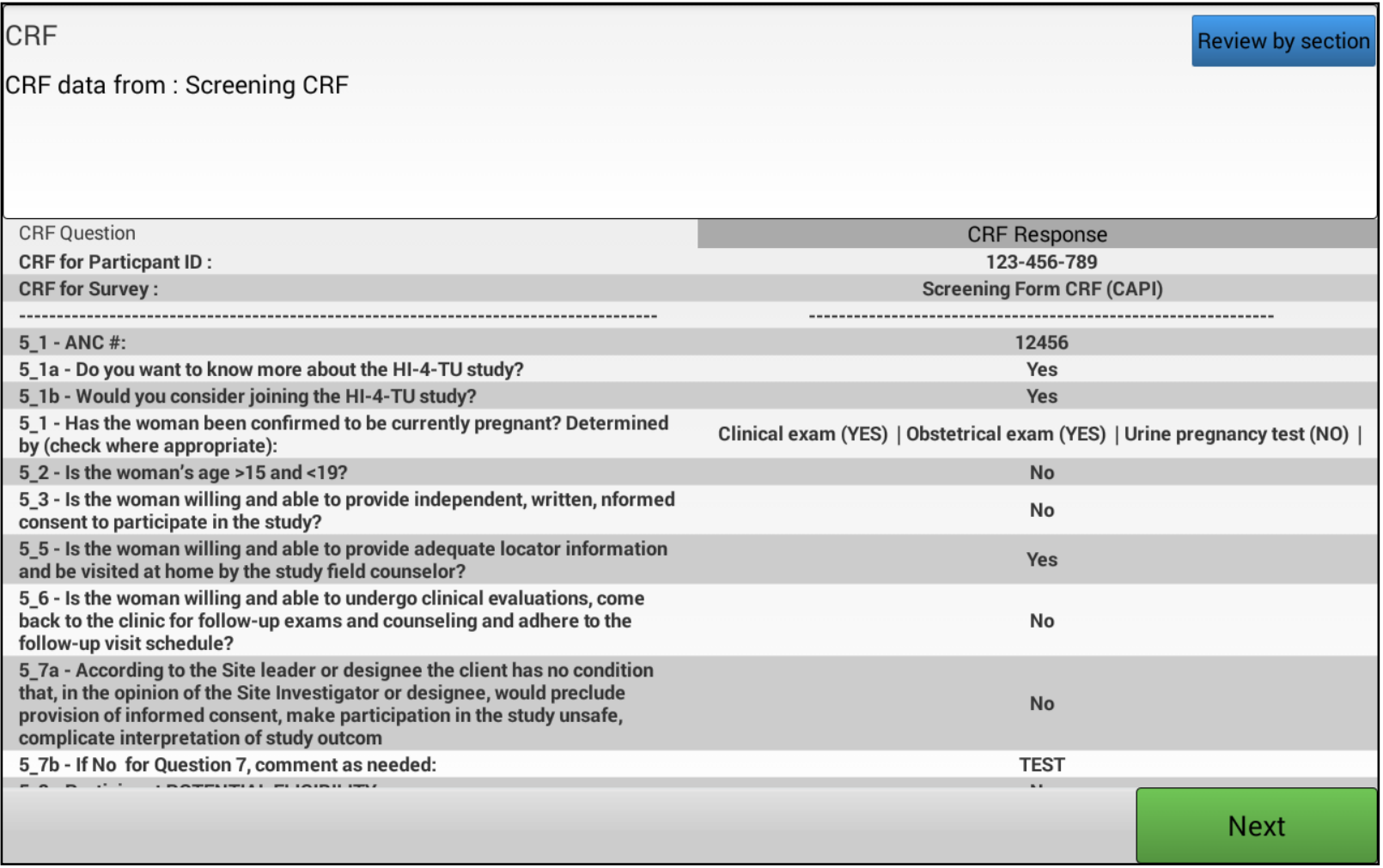

Fig. 4 Example e-CRF screen integrated into PC ACASI.

The e-CRF form completion was accomplished by utilizing the Android devices' touchscreen capabilities, and each question was displayed individually. Pre-built logic was designed and integrated into the e-CRF where necessary, and response options were limited and restricted to minimize data entry error. A check list of necessary criteria for participation was provided to the clinic staff at the site, as seen in Figure 4 and 5. This allowed the site staff to ensure the data was accurate before guiding the participant inside the clinic for flow for the study.

\section{ACASI + CRF Designed Solution - Technical}

The base Android tablet data collection software solution was written using the Java programming language, complemented with the SQLite database. All software development was done using the open source Eclipse integrated development environment (IDE). The data collection system permits for data to be collected offline with the Android tablet devices, and to merge with a source data manager laptop computer, without the need for Internet connectivity. When Internet connectivity is available to the data manager computer, the solution provides for the ability to upload the data to a secure web site or server [2]. This functionality was built using the Microsoft Azure cloud and also permitted for software updates to be made to each Android device when connected to the Internet. 


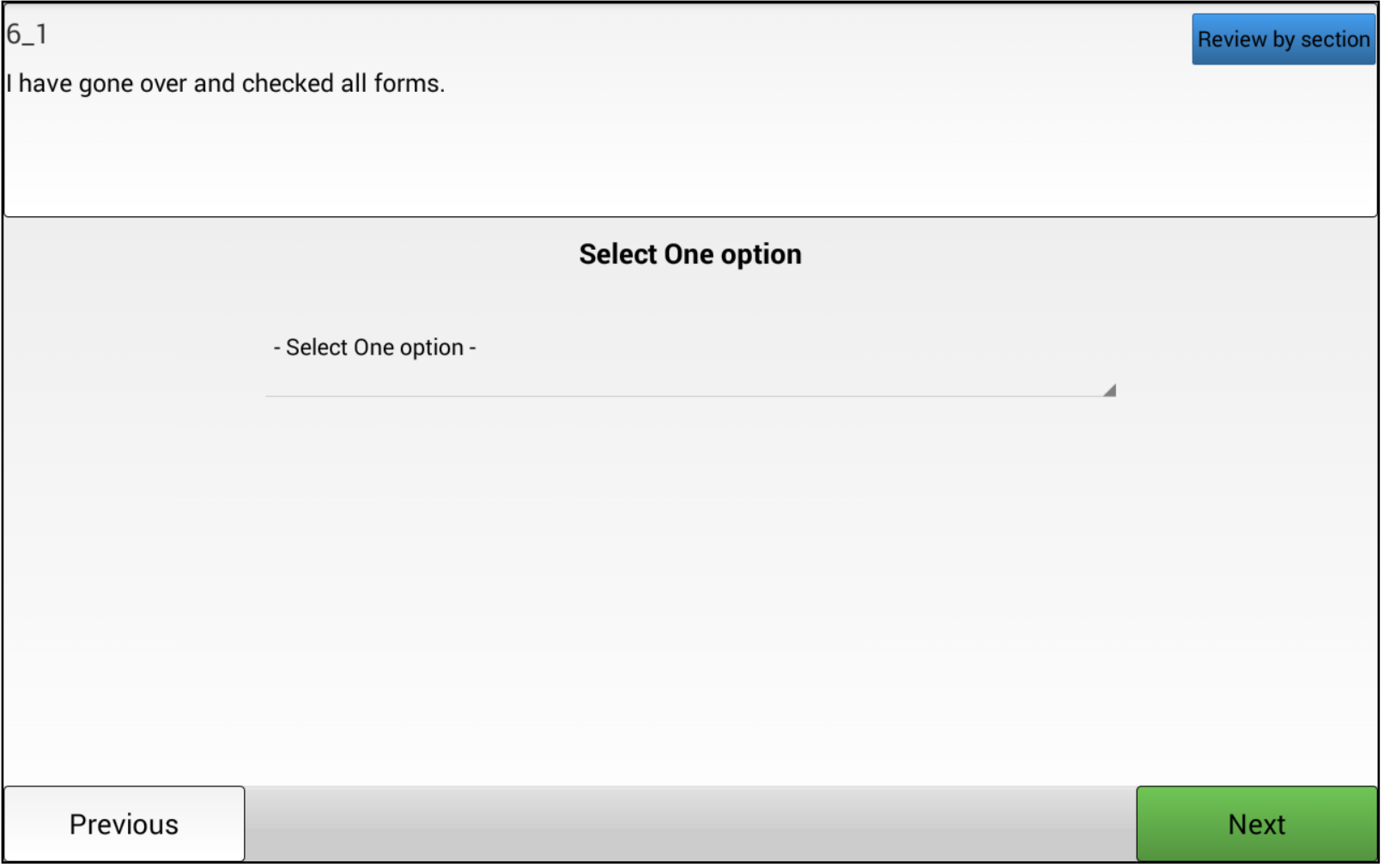

Fig. 5 e-CRF Response Review and Confirmation.

\section{Discussion}

Automating paper $\mathrm{CRF}$ processes to an electronic format can introduce certain new issues that should be considered. One new requirement for future like research efforts will be to involve the Information Technology departments in the study sites to provide technology support. Paper-based CRF did not directly require technology assistance from either the installation or setup perspective or from the technical support perspective. This can introduce delays if clear goals and needs are not properly communicated to the Information Technology unit as well as receiving a commitment from the unit [3].

Electronic CRF's continue to be the normal process for collecting and managing data in clinical trials. The use of paper CRF's or use of fax and optical character recognition have been used frequently in locations in Sub-Saharan Africa for many years, but are now being replaced with such cloud-enabled tools as Medidata solutions, specifically the RAVE module. These new breed of electronic systems for clinical trial data management are proving effective in Sub-Saharan Africa especially as the Internet infrastructure has continued to be more and more reliable and effective. However, these new tools may not include a way to integrate more innovative and novel ways of collecting data with a semi-literate population, traditionally when self-report modality of surveying is required for more sensitive questions of nature. There would be a benefit to introduce ACASI, CASI, V-ACASI into these new electronic systems for clinical trial data management. Although the ACASI type of module or survey modality may not be needed for all participants or trial efforts, there still remains a need for use as was exhibited in this paper. Vendors of validated 
clinical trial data collection tools should continue to consider integrating more novel or custom ways of integrating innovative self-report survey techniques, such as ACASI into their toolsets. Questionnaire design still does remain as much an art as a science, but the evidence base for improving the quality and completeness of data collection in clinical trials is growing [4].

\section{Conclusion}

During the process of developing automated systems, engineers and software developers should consider how to "future proof" the solutions. This would involve considering how to make a solution stay useful over time and continue to be valuable. As of the time of this publication successful data collection has gone on for nineteen months. During this timeframe 694 screening and enrollment ACASI surveys have been conducted and 340 follow-up surveys. E-CRFs will continue to gain use in the developing world, which makes sense because automating the process helps to minimize errors that can be introduced with completing paper forms or transposing the paper results to an electronic record. Differences in data entry methods can affect the trial in terms of data accuracy, cost, and efficiency. For example, when compared with paper case report forms, electronic data capture can reduce the time required for data entry, query resolution, and database release or study data closure by combining data entry with data collection [5] [3]. Integrating or adding ACASI to the e-CRF solution will help to minimize disparate systems and will be particularly valuable when there is a need to capture self-report data, such as measuring adherence and acceptability. Cost savings can be obtained if there is an ability to integrate self-report data collection into an already existing e-CRF solution. Self-report survey data integration with e-CRF is not limited to ACASI; it can also include other technical solutions such as Interactive Voice Response Systems (IVRS), Computer-Assisted Telephone Interviews (CATI), Computer-Assisted Self-Interviews or Short Message Service (SMS) where data is collected.

\section{Limitations}

The technology solution developed for HI-4-TU was not validated for use in clinical trials. Data collection that will be utilized as part of an effort for submission to health regulatory bodies, such as the US Food and Drug Administration (FDA), will require that such systems be compliant with 21 CFR Part 11. We did not evaluate the costs associated with migrating from paper-based CRF to e-CRF, but future research would be beneficial to the community to include this complementary information. There are advantages with data quality and monitoring that come with e-CRF, but the cost benefit could be a function of the size of the trial - meaning that the larger the number of trial participants as well as the number of sites, the greater the potential savings.

\section{Acknowledgements}

The authors would like to thank the project sponsor from Johns Hopkins University as well as the collaborators from Makerere University - Johns Hopkins University (MU-JHU) Research Collaboration and the Population Council. We would like to acknowledge Irene Friedland, at the Population Council, for her contributions to the thorough edit she has provided to this paper. 


\section{References}

1. Kamala Thriemer; Benedikt Ley; Shaali M Ame; et al. 2012. Replacing paper data collection forms with electronic data entry in the field: findings from a study of communityacquired bloodstream infections in Pema, Zanzibar. BMC Res Notes. 20125, 113. PubMed https://doi.org/10.1186/1756-0500-5-113

2. Kathryn L Falb, ScD; Sophie Tanner; Khudejha Asghar; Samir Souidi; Stan Mierzwa et al. 2017. Implementation of Audio-Computer Assisted Self-Interview (ACASI) among adolescent girls in humanitarian settings: Feasibility, acceptability, and lessons learned. Confl Health. 10, 32. PubMed

3. Welker JA. 2006. Implementation of electronic data capture systems: Barriers and solutions. Contemp Clin Trials. 28(3): 329-36. PubMed

4. Edwards P. 2010. Questionnaires in clinical trials: guidelines for optimal design and administration. BioMed Central Trials. 11, 2. PubMed

5. Litchfield J, Freeman J, Schou H, et al. 2005. Is the future of clinical trials internet-=based? A cluster randomized clinical trial? A cluster randomised clinical trial. Clin Trials. 2, 72-79. PubMed https://doi.org/10.1191/1740774505cn069oa

6. Guidelines for Clinical Research. World Health Organization, http://apps.who.int/medicinedocs/en/d/Jh2948e/6.8.html

7. Maarten A Wildeman; Jeroen Zandbergen; Andrew Vincent; et al. 2011. Can an online clinical data management service help in improving data collection and data quality in a developing country setting? Trials. 12, 190. PubMed https://doi.org/10.1186/1745-6215-12$\underline{190}$

8. Maarten A. 2011. Wildeman, Jeroen Zandbergen, Andrew Vincent, etc. al "Can an online clinical data management service help in improving data collection and data quality in a developing country setting?". Trials. 12, 190. PubMed https://doi.org/10.1186/1745-6215$\underline{12-190}$ 